\title{
Glassy Behavior of a Tin Dioxide Nanoparticle Suspension
}

\author{
Atsumi Wakabayashi, ${ }^{\dagger}$ Tatsuhiko Goto, ${ }^{\ddagger}$ Toshiaki Dobashi, ${ }^{\ddagger}$ Yasuyuki Maki ${ }^{* \ddagger}$
}

${ }^{\dagger}$ Advanced Materials Division, Sumitomo Osaka Cement Company, Ltd., Funabashi,

Chiba 274-8601, Japan

${ }^{\ddagger}$ Division of Molecular Science, Faculty of Science and Technology, Gunma University, Kiryu, Gunma 376-8515, Japan

email: maki@gunma-u.ac.jp

\section{SUPPORTING INFORMATION}

Transmission Electron Microscope (TEM) Observation. Transmission electron micrographs were recorded with a JEOL JEM-2010 transmission electron microscope (TEM) (Figure S1). Samples for the TEM observation were prepared by spotting grids with a $\mathrm{SnO}_{2}$ suspension with a concentration of $0.1 \mathrm{wt} \%\left(\varphi=1.4 \times 10^{-4}\right)$ and letting the spots dry.

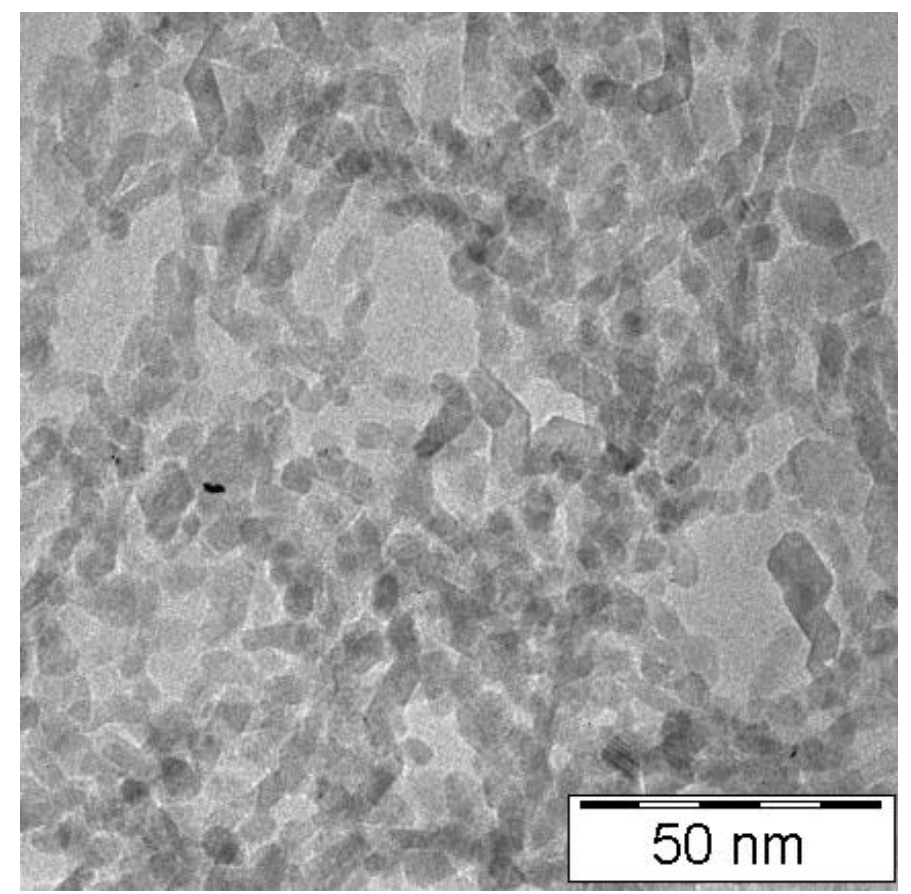

Figure S1. TEM image of $\mathrm{SnO}_{2}$ nanoparticles. 
Estimation of the Debye Length from the Conductivity Experiment. The Debye length $\kappa^{-1}$ is represented as

$$
1=\sqrt{\frac{k T}{2 N_{\mathrm{A}} e^{2} I}} .
$$

Here, $\varepsilon, N_{\mathrm{A}}$, and $I$ are the dielectric constant of the medium, Avogadro's number, and the ionic strength, respectively. The ionic strength is defined as $I=(1 / 2) \sum Z_{i}^{2} n_{i}$, where $Z_{i}$ and $n_{i}$ are the valence and molar concentration of $i$ th ion, respectively. The concentration $n_{i}$ of $i$ th ion in colloidal suspensions can be estimated from the conductivity $\sigma$ by use of the equation $\sigma=\sum \mu_{i} Z_{i} n_{i} e$, where $\mu_{i}$ is the mobility of $i$ th ion. For the $\mathrm{SnO}_{2}$ nanoparticle suspension of $1.0 \mathrm{wt} \%\left(\varphi=1.4 \times 10^{-3}\right), \sigma$ was measured to be $2.5 \mathrm{mS} / \mathrm{m}$. Assuming that the mobile ions in the suspension are residual ammonium ions and chloride ions, and using $\mathrm{NH}_{4}=7.63 \times 10^{-8} \mathrm{~m}^{2} \mathrm{~s}^{-1} \mathrm{~V}^{-1}$ and $\quad \mathrm{Cl}_{1}=7.91 \times 10^{-8} \mathrm{~m}^{2} \mathrm{~s}^{-1} \mathrm{~V}^{-1}, \kappa^{-1}$ was calculated to be approximately $5 \mathrm{~nm}$ for the suspension with a concentration of $25 \mathrm{wt} \%(\varphi=0.045)$. 